\title{
ESTIMADOR BORROSO DE UNA PLANTA SOLAR CILINDRO-PARABÓLICA
}

\author{
Adolfo J. Sánchez \\ Universidad de Sevilla \\ asanchezdelpozo@us.es
}

\author{
Juan Manuel Escaño \\ Universidad Loyola Andalucía \\ jmescano@uloyola.es
}

\author{
Carlos Bordons \\ Universidad de Sevilla \\ bordons@us.es
}

\author{
Eduardo F. Camacho \\ Universidad de Sevilla \\ efcamacho@us.es
}

\section{Resumen}

La estimación de los estados no observables de un proceso es importante cuando se usan técnicas de control que suponen dichos valores conocidos apriori. Los controladores basados en el espacio de estados presentan un buen comportamiento y eficiencia, aún cuando la dinámica del proceso es no lineal. Uno de los procesos con una dinamica no lineal y en la que no todos los estados son observables es el caso de las plantas solares cilindroparabólicas. En este trabajo se presenta un observador, basado en un sistema de inferencia borroso, para la estimación de los perfiles de temperatura de los lazos que componen el campo solar.

Palabras clave: Estimador borroso; Planta cilindro-parabólica; Control basado en modelo

\section{INTRODUCCION}

En el control automático de procesos industriales, cuanta más información sobre el proceso tenga el controlador que se ha diseñado, mejor será el control del mismo.

La información que se podrá proporcionar al controlador, dependerá en todo caso del tipo de controlador que se ha diseñado. En el caso de controladores clasicos como un control Proporcional Integral Derivativo (PID) la única información que recibirá es el error entre la referencia y la salida del sistema a controlar. Por lo que el conocimiento del estado de la planta en el controlador se limita a una señal de error. En otro tipo de controladores más complejos, la información que se le podrá proporcionar es mayor. El hecho de utilizar controladores más complejos puede ser debido a que la dinámica del sistema es muy no lineal. En particular, algunos métodos de control asumen que el estado del proceso a controlar es conocido. Dentro de estos podemos encontrar que las técnicas de Model Predictive Control (MPC) se han hecho muy populares y eficientes. Los MPC diseñados en el espacio de estados necesitan conocer el valor de los diferentes estados del sistema. Estos estados pueden ser o no observables, es decir, si las variables de estado se pueden medir o no. $\mathrm{Y}$ en caso de no poder medirse por imposibilidad física o incluso económica pueden estimarse y el controlador puede usar las estimaciones como si fueran medidas.

Un caso particular donde no todos los estados pueden ser medidos, por motivos principalmente económicos, es una planta solar cilindroparabólica. Estas plantas están formadas por numerosos lazos de captadores solares de cientos de metros $[9,13,15,16]$. Dada la naturaleza no lineal del proceso del campo solar se han diseñado multiples técnicas de control MPC en el espacio de estados obteniendo bueno resultados. En [6], los autores proponen un modelo adaptativo MPC en el espacio de estados para el control del campo solar con una estimación mediante Unscented Kalman Filter (UKF). Un MPC más avanzado en el espacio de estados se presenta en [7] donde se propone un observador de Luenberguer para la estimación de los estados. Un Extended Kalman Filter (EKF) se propone en [12] para la estimación de las temperaturas de internas del lazo y de entrada suponiendo que no existe un sensor a la entrada y su aplicación en un PID. En [14], los autores proponen una optimización no lineal para controlar las valvulas de entrada y una estimación de los estados mediante el uso de Classification and Regression Trees (CARTs) necesaria para la optimización basada en un modelo no lineal reducido.

Los estimadores lineales, como el observador de luenberguer, están basados en el modelo lineal de la planta que no es más que una aproximación del modelo no lineal. Por tanto, se esperan errores de predicción en la estimación de los estados. Por otro lado, estimadores basados en datos como en el caso de los CARTs, pueden llegar a proporcionar valores pequeños del error si están bien diseñados aunque a un coste computacional mayor debido al tamaño que ocupará en memoria el estimador. Aunque, generalmente, un pequeño error no es preocupante en el control, la idea es que sea el menor posible y con el menor coste computacional posible.

En este trabajo trata sobre la estimación de los estados del campo solar basado en Fuzzy Logic. 
Los sistemas de inferencia borrosa o Fuzzy Inference Systems (FIS) son aproximaciones funcionales genéricas, es decir, dado un cierto nivel de error, es posible encontrar un FIS que aproxima cualquier función con un error menor que el fijado. Esto hace que el FIS sea adecuado para $\mathrm{su}$ uso como estimadores, incluso no tienen que estar basados en el espacio de estados necesariamente. Se pueden encontrar muchos métodos en la literatura para la identificación de un FIS. Uno de los métodos más populares es la formulación de un sistema borroso tipo Takagi-Sugeno (TS) [17] como una Red Neural o Neural Network (NN) [11], también llamado Sistema de Inferencia Neuro-borroso Adaptativo (ANFIS). Una de las formas clásicas de modelar sistemas complejos experimentalmente es mediante el uso de NN artificial. Un FIS utilizado para la estimación, puede manejar sistemas no lineales y proporcionar conocimiento del sistema que es imposible con el uso de NN. En este trabajo se propone un sistema borroso tipo TS con funciones de pertenencias gausianas, del tipo:

$$
m(x ; c, \sigma)=e^{-\frac{1}{2}\left(\frac{x-c}{\sigma}\right)^{2}}
$$

Y con un conjunto de reglas: $R_{j}$ :

IF $x_{1}(k)$ is $F_{1 j}$ and $x_{2}(k)$ is $F_{2 j}$, THEN:

$y_{j}(k)=a_{1 j} x_{1}(k)+a_{2 j} x_{2}(k)+a_{3 j}$, donde $a_{i j} \in \mathbb{R}$

Existen numerosos algoritmos aplicables a ANFIS que aprenden a actualizar los parámetros de las capas. Notablemente, por ejemplo, Backpropagation (BP) [18] usado mucho en NN. Existen otros métodos combinados con BP, como la combinación híbrida de mínimos cuadrados y la retropropagación, [11], [8]. Una de las ventajas de los sistemas borrosos frente a las NN es la adición de reglas. Estas reglas vienen dadas por el conocimiento experto y la observación del sistema por parte del ingeniero. Como se discutió anteriormente, una de las propiedades más importantes del FIS es su capacidad para aproximar funciones no lineales con error de aproximación acotada. Mejorar la precisión causa un aumento en el número de reglas. Se debe considerar que un sistema basado en demasiadas reglas no es práctico y causa una pérdida de comprensión del sistema. El uso de un método de agrupamiento puede evitar la explosión de reglas y obtener los clústeres naturales entre las variables de entrada y salida. En esta sección, se presentan algunas técnicas populares y ampliamente utilizadas para obtener la estructura y capacitar al FIS para que se ajusten a los datos reales.

En articulo está organizado como sigue: En la sección 2 se presenta el modelo del campo solar Acurex y los modelos matemáticos que se utilizan en este trabajo. En la Sección 3 se presenta la obtención del estimador borroso de un lazo del campo Acurex. En la Sección 4 se muestran los resultados obtenidos de la estimación borrosa en dos escenarios distintos simulados y en la Sección 5 se finaliza con unas conclusiones.

\section{CAMPO SOLAR ACUREX}

El campo ACUREX, ubicado en la Plataforma Solar de Almería, es un campo solar de 480 captadores cilindro-parabólicos. Los colectores están dispuestos en 10 lazos. La longitud total de cada lazo es de $172 \mathrm{~m}$, que comprende partes activas (142 m) y partes pasivas, es decir, uniones y otras partes a las que no alcanza la radiación solar concentrada $(30 \mathrm{~m})$.

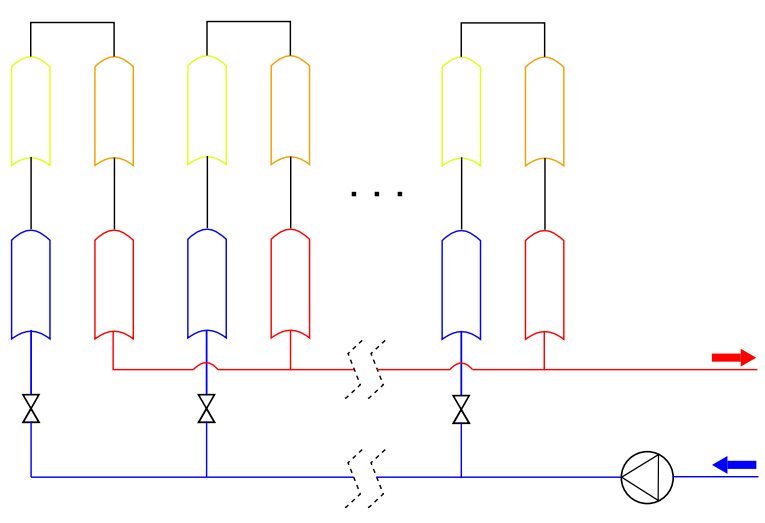

Figura 1: Acurex Solar Field schematic.

En este trabajo se utilizan dos tipos de modelos matemáticos para la planta: un modelo de parámetros distribuidos y un modelo de parámetros concentrados. Ambos modelos se han obtenido a través de pruebas y validaciones realizadas en la planta y han sido utilizados por muchos autores. Un esquema de la planta se muestra en la figura 1. Para una descripción completa del procedimiento de modelado de la planta, consul$\operatorname{tar}[2,3]$.

\subsection{Modelo de parámetros distribuidos}

La dinámica del campo del colector solar distribuido se describe mediante el siguiente sistema de ecuaciones diferenciales parciales (PDE) que describen el balance de energía que se produce en el lazo $[2,3]$ : 


$$
\begin{aligned}
\rho_{m} C_{m} A_{m} \frac{\partial T_{m}}{\partial t} & =I K_{o p t} n_{o} G-H_{l} G\left(T_{m}-T_{a}\right) \\
& -L H_{t}\left(T_{m}-T_{f}\right)
\end{aligned}
$$

$$
\rho_{f} C_{f} A_{f} \frac{\partial T_{f}}{\partial t}+\rho_{f} C_{f} \dot{q} \frac{\partial T_{f}}{\partial x}=L H_{t}\left(T_{m}-T_{f}\right)
$$

donde el subíndice $m$ se refiere al metal y $f$ se refiere al fluido. Los parámetros y unidades del modelo se muestran en la Tabla 1.

Tabla 1:

Descripción de parámetros.

\begin{tabular}{lll}
\hline Símbolo & Descripción & Unidad \\
\hline$t$ & Tiempo & $\mathrm{s}$ \\
$x$ & Espacio & $\mathrm{m}$ \\
$\rho$ & Densidad & $\mathrm{kg} / \mathrm{m}^{3}$ \\
$C$ & Capacidad calorífica especifica & $\mathrm{J} /\left(\mathrm{kg}{ }^{\circ} \mathrm{C}\right)$ \\
$A$ & Área de la sección transversal & $\mathrm{m}^{2}$ \\
$T$ & Temperatura & ${ }^{\circ} \mathrm{C}$ \\
$T_{a}$ & Temperatura Ambiente & ${ }^{\circ} \mathrm{C}$ \\
$q$ & Caudal de aceite & $\mathrm{m}^{3} / \mathrm{s}$ \\
$I$ & Radiación Solar & $\mathrm{W} / \mathrm{m}^{2}$ \\
$n_{o}$ & Eficiency Geométrica & Adimensional \\
$K_{o p t}$ & Eficiency Óptica & Adimensional \\
$G$ & Apertura del Colector & $\mathrm{m}$ \\
$H_{l}$ & Coeficiente de perdidas térmicas & $\mathrm{W} /\left(\mathrm{m}^{2 \circ} \mathrm{C}\right)$ \\
$H_{t}$ & Coeficiente de transmision de calor metal-fuido & $\mathrm{W} /\left(\mathrm{m}^{2 \circ} \mathrm{C}\right)$ \\
$L$ & Longitud de la tubería & $\mathrm{m}$ \\
\hline & &
\end{tabular}

La eficiencia geométrica depende del ángulo horario, la hora solar, la declinación, el día del año, la latitud local y las dimensiones del colector. La densidad $\rho$, el calor específico $C$ y el coeficiente de transmisión de calor $H_{t}$ dependen de la temperatura del fluido. El coeficiente de transmisión de calor depende de la temperatura y del flujo de aceite [2]. El modelo está discretizado en la dimensión longitudinal del tubo por lo que la dinámica de cada lazo se puede simular como una cadena de submodelos. Se ha elegido una longitud de segmento de 1 metro para la implementación del modelo de simulación (172 segmentos por lazo).

\subsection{Modelo de parámetros concentrados}

El modelo de parámetros concentrados proporciona una descripción general de todo el campo. La variación en la energía interna del fluido se puede describir mediante la ecuación:

$$
\begin{aligned}
C \frac{d T_{\text {out }}}{d t} & =K_{\text {opt }} n_{o} S I-q P_{c p}\left(T_{\text {out }}-T_{\text {in }}\right) \\
& -H_{l}\left(T_{m}-T_{a}\right)
\end{aligned}
$$

donde $C$ es la capacidad térmica del lazo, $K_{o p t}$ es la eficiencia óptica que tiene en cuenta elementos como la reflectividad y la absortancia. La eficiencia geométrica $n_{o}$ está determinada por la posición de los espejos respecto del vector del haz de radiación. El factor $P_{c p}$ tiene en cuenta algunas propiedades geométricas y térmicas del ciclo. $T_{i n}$ es la temperatura de entrada, $H_{l}$ es el coeficiente de pérdidas térmicas globales, $T_{m}$ es la temperatura media entre la temperatura de entrada y salida, y $T_{a}$ es la temperatura ambiente. El coeficiente $H_{l}$ se puede aproximar por $1.05 \mathrm{~kW} /{ }^{\circ} \mathrm{C}$, $P_{c p}$ por $1.924 \times 10^{6} \mathrm{~kJ} /{ }^{\circ} \mathrm{C}, \mathrm{C}$ por $2267 \mathrm{~kW} /{ }^{\circ} \mathrm{C}$, y la superficie total reflectante $S$ toma el valor de $2674 \mathrm{~m}^{2}$. Estos valores se han obtenido en base a los datos relativos a las medidas en la planta que funcionan en diferentes puntos de ajuste mediante el análisis de regresión múltiple [2].

\section{ESTIMADOR BORROSO DE TEMPERATURA DE SEGMENTO}

Para el entrenamiento del sistema borroso es necesario disponer de un conjunto amplio de datos. Este conjunto de datos se dividirá en dos partes: una primera parte se dedicará al entrenamiento del estimador y la segunda parte servirá para hacer el test de chequeo para comprobar el error obtenido con el sistema diseñado. El set de datos se creará utilizando el modelo de parámetros distribuidos. Se simulará el campo solar modificando los valores de irradiancia, eficiencia geométrica, temperatura ambiente y caudal de aceite. Dado que no es computacionalmente viable tener un conjunto de datos que contengan toda la dinámica no lineal de la planta en cualquier punto de operación, el conjunto de datos se limitará a la obtención de las variables en el régimen permanente. Una vez la planta llegue al régimen permanente se obtendrá el valor de la temperatura de salida del lazo así como los valores de temperatura de cada trozo de tubería. Aunque el conjunto de datos incluyen valores para la irradiancia, eficiencia geométrica, etc, para el entrenamiento se ha optado por utilizar solamente la temperatura de entrada, de salida y el caudal como entradas y las temperaturas de los diferentes segmentos del lazo como salida. Como se puede intuir, básicamente el estimador se entrenará en base al salto térmico que se produce en el lazo y un caudal de aceite. De esta forma dado un caudal y un salto térmico, se podrá estimar la temperatura de cada trozo. Se ha optado esta forma de entrenar el sistema para reducir el conjunto de datos y evitar la explosión de reglas dado que, al fin y al cabo, un salto térmico $\mathrm{y}$ un caudal determinado pueden equivaler, por ejemplo, tanto a una irradiancia mayor y una eficiencia geométrica menor como a una irradiancia 
menor y una eficiencia geométrica mayor. En la obtención del estimador y por motivos computacionales, tanto en el tamaño del estimador borroso como para su posterior uso, por ejemplo, en MPCs en el espacio de estados, el lazo se dividirá en 16 trozos en lugar de los 172 trozos en los que se divide el modelo de parámetros distribuidos. La estimación borrosa de cada una de las temperaturas de los 16 segmentos se realiza con el mismo tiempo de muestreo que se utiliza en el controlador de la planta, generalmente, $T_{s}=39 \mathrm{~s},[2]$.

Inicialmente, para obtener las funciones de pertenencia, es útil usar un método de agrupamiento. Muchos algoritmos salen para el análisis de clustering [5], [10], [1], [19]. Un algoritmo rápido de una sola iteración para estimar el número de agrupaciones y los centros de las mismas de un conjunto de datos es la Agrupación sustractiva (Subtractive Clustering, SC) [4]. Esta técnica, como cualquier método de agrupamiento, se usa para obtener las variables lingüísticas apropiadas. El método SC es una modificación del Método de la montaña [20]. En SC, se define la función

$$
M_{i}=\sum_{j=1}^{N} e^{-\alpha\left\|z_{i}-z_{j}\right\|^{2}}
$$

llamada función de la montaña, donde $\alpha=4 / r_{a}^{2} \mathrm{y}$ $r_{a}>0$ define el radio de vecindad para cada grupo (se elige según la resolución deseada para grupos). Si $F_{1}^{*}$ es el mayor valor de la función, perteneciente a el punto $z_{1}^{*}$, escogido como el centro del grupo, para cada punto $z_{i}$ se calcula la siguiente función reducida

$$
M_{i} \Longleftarrow M_{i}-M_{1}^{*} e^{-\beta\left\|z_{i}-z_{1}^{*}\right\|^{2}}
$$

El algoritmo es el siguiente:

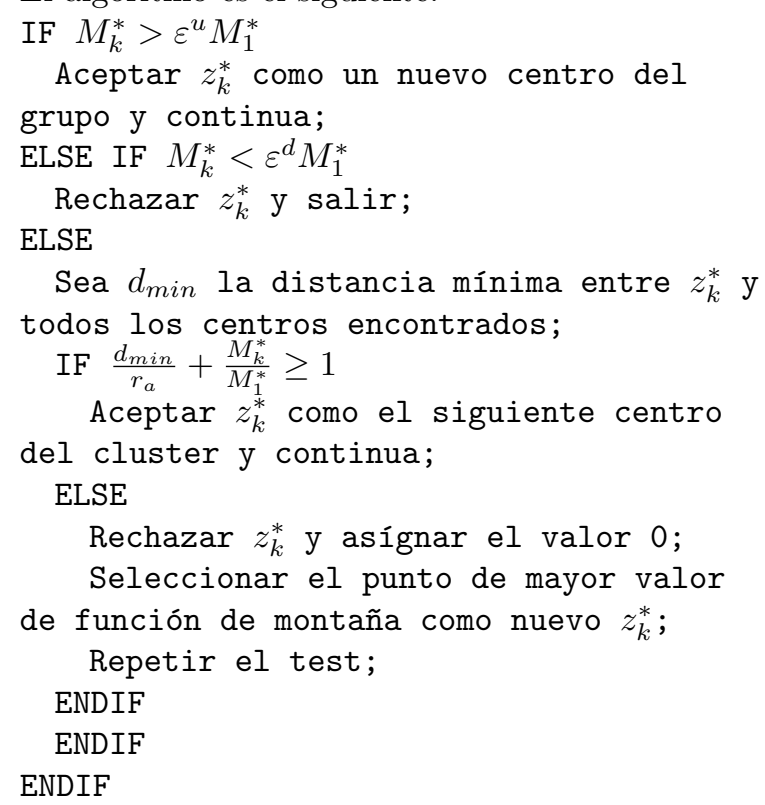

Donde $\varepsilon^{u}$ es un umbral por encima del cual, el punto se selecciona como centro y $\varepsilon^{d}$ otro umbral por debajo del cual el punto se desecha. Típicamente $\varepsilon^{u}=0.5$ y $\varepsilon^{d}=0.15$ (con valores normalizados). El radio para el potencial reducido debe de ser un grado mayor que el radio del vecino para prevenir agrupaciones espaciadas. Normalmente $r_{b}=1.5 \cdot r_{a}$. En la figura 2 se presentan las funciones de pertenencia resultantes para para cada entrada (salto térmico y caudal) y cada segmento. Se puede observar similaridades entre las funciones de cada lazo.
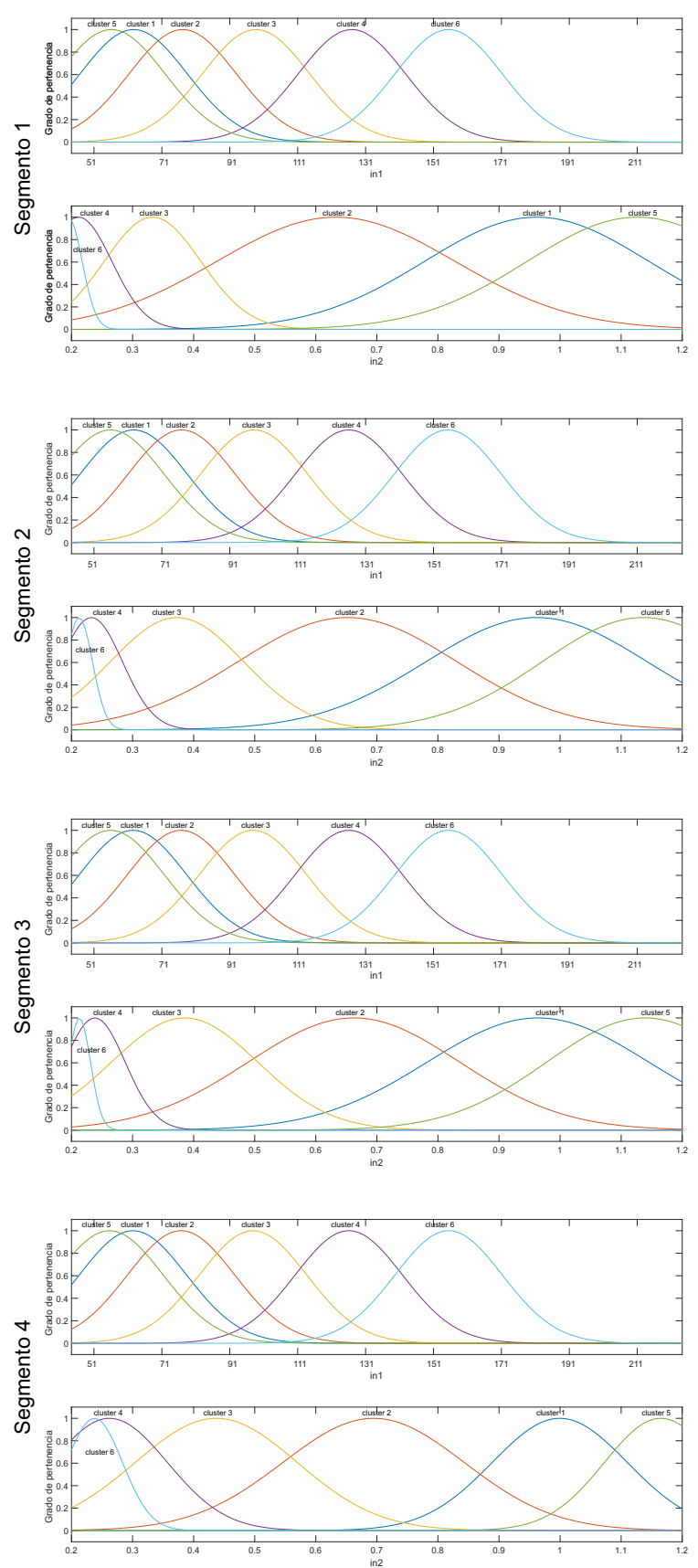

Figura 2: Funciones de pertenencia de los estimadores borrosos 

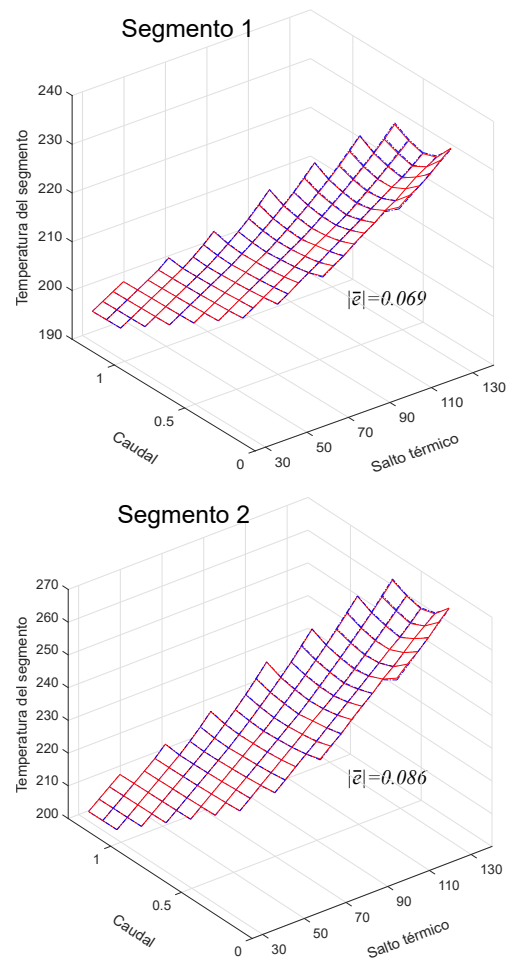

Segmento 3

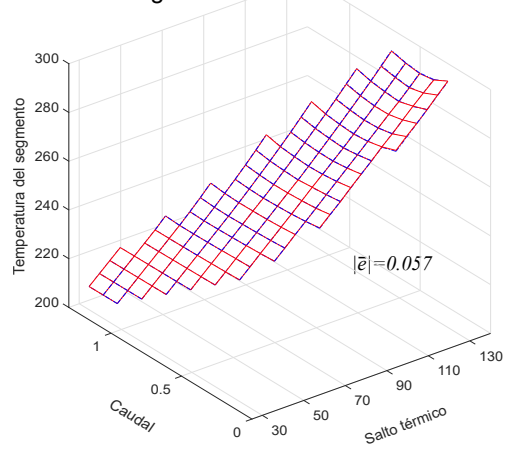

Segmento 4

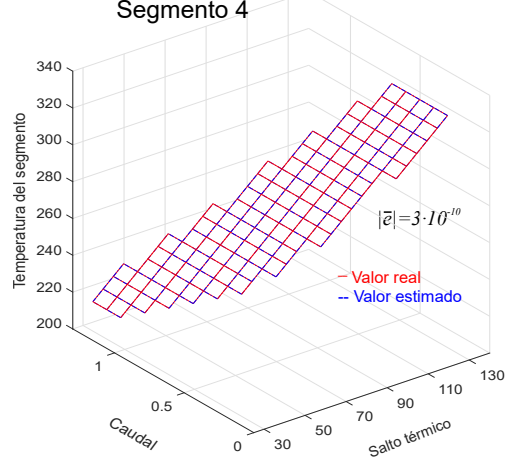

Figura 3: Temperatura de salida real y estimada por cada segmento $\left({ }^{\circ} \mathrm{C}\right)$. Entradas: Salto térmico $\left({ }^{\circ} \mathrm{C}\right)$ y caudal $\left(\mathrm{m}^{3} / \mathrm{s}\right)$

En la figura 3 se representan las superficies de los estimadores, frente a los valores medidos de temperatura en cada segmento, junto a una medida de validación, tal y como la media del valor absoluto del error de estimación: $\overline{|e|}$. Se puede observar que el último lazo, al estar muy cercano a la tem- peratura de salida, posee un error prácticamente nulo.

\section{RESULTADOS}

En esta sección se presentan los resultados de las estimaciones de las temperaturas de los segmentos de distintos lazos.

Acurex es una planta que consta de 10 lazos que pueden tener diferentes reflectividades debido a la suciedad. Las simulaciones se han llevado a cabo aplicando un controlador basado en modelo cuya misión será seguir una referencia de la temperatura de salida del campo. Dado que la planta consta de diez lazos, la temperatura de salida del campo será una media ponderada de las temperaturas de todos los lazos que componen el campo.

En las simulaciones se aplican cambios en los setpoints de temperatura de salida de campo para cambiar el punto de trabajo de la planta y observar el funcionamiento de la estimación en diferentes puntos de operación. Se han simulados dos casos en los que se presentan simulaciones con diferentes set-points de temperatura a lo largo del día. En estas simulaciones cada lazo tiene una reflectividad diferente, ver Tabla 2.

Tabla 2:

Casos Simulados.

\begin{tabular}{lllllllllll}
\hline Set de Reflectividades & L1 & L2 & L3 & L4 & L5 & L6 & L7 & L8 & L9 & L10 \\
\hline Caso 1 & 0.65 & 0.67 & 0.68 & 0.7 & 0.715 & 0.73 & 0.75 & 0.77 & 0.79 & 0.8 \\
Caso 2 & 0.67 & 0.71 & 0.71 & 0.74 & 0.74 & 0.76 & 0.76 & 0.79 & 0.79 & 0.8 \\
\hline
\end{tabular}

Por motivos visuales y de espacio se presentan los resultados de dos de los diez lazos con distintas reflectividades y se presentan las estimaciones de los 4 segmentos que corresponden a cada uno de los 4 colectores que componen un lazo.

El primer escenario simulado se presenta en las figuras 4,5 y 6 .

En la figura 4 se presenta la simulación del funcionamiento del controlador basado en modelo del caso 1 . Se puede observar como consigue mantener un buen seguimiento de la referencia aplicada en la temperatura de salida de campo.

En la figura 5 se muestra la estimación borrosa de las temperaturas de los segmentos del lazo 1, con una reflectividad 0.65. Es posible observar que el estimador tiene un buen comportamiento tanto en dinámico como en estático. 

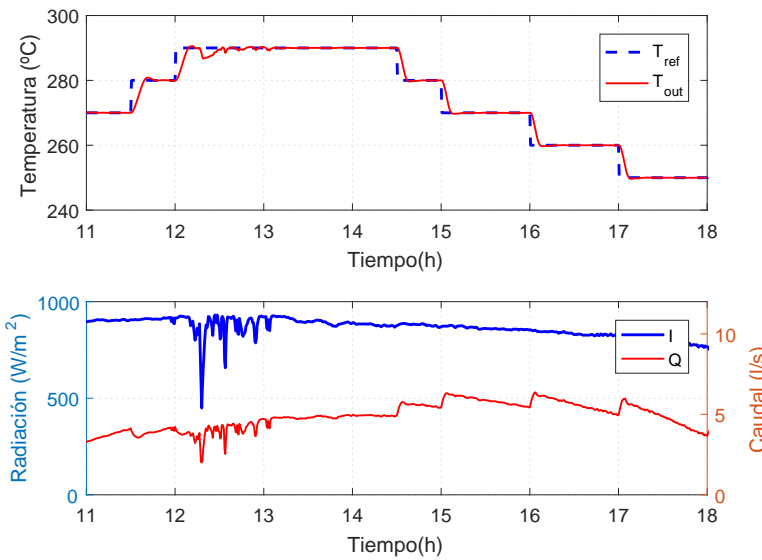

Figura 4: Simulación Caso 1. Seguimiento de setpoint de temperatura de salida de campo.
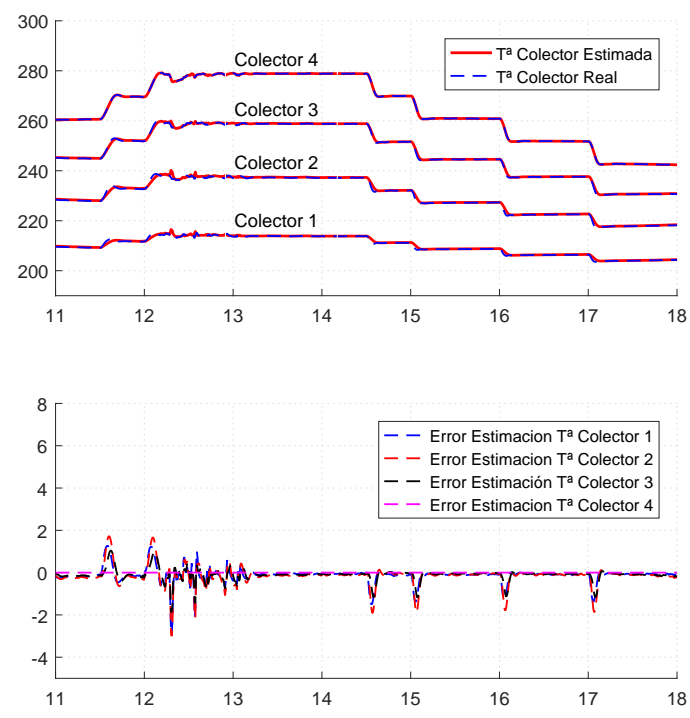

Figura 5: Simulación Caso 1. Etimación de temperaturas de 4 segmentos del Lazo 1 con reflectivi$\operatorname{dad} 0.65$.

En la figura 6 se presenta la estimación borrosa de las temperaturas de los segmentos del lazo 10, con una reflectividad 0.8 . Se observa que, aunque teniendo una reflectividad mayor, el estimador es capaz de captarlo pues se está utilizando la temperatura de salida y entrada de lazo como entrada al sistema borroso. Como se explicó anteriormente, al diseñar el estimador se utilizaron datos en régimen permantente de la planta con variaciones en la radiació, caudal, temperatura ambiente, etc. Dado que solo se utilizaron temperaturas y caudal en el entrenamiento, la reflectividad viene reflejada implicitamente en los incrementos de temperatura que se producen en el lazo entre la entrada y la salida. Es por esto que la estimación es satisfactoria en lazos con diferentes reflectividades.
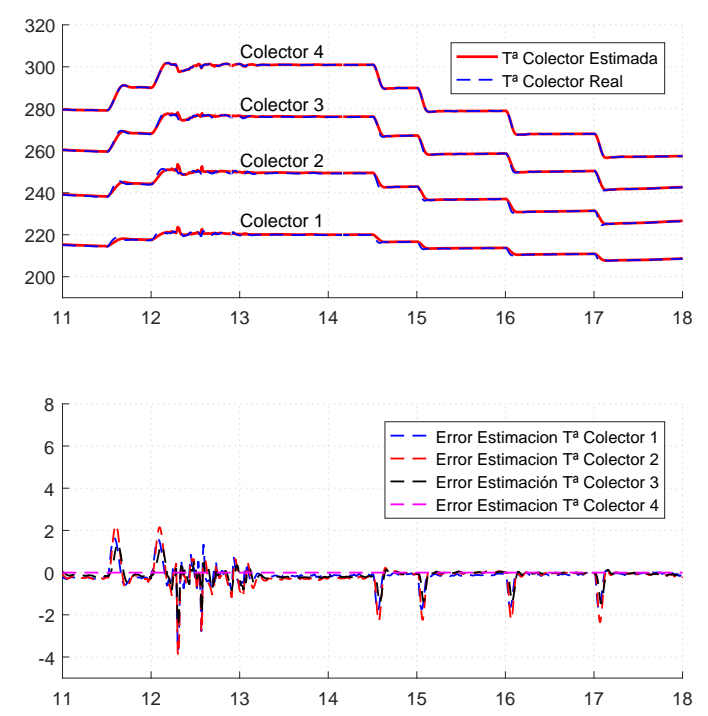

Figura 6: Simulación Caso 1. Etimación de temperaturas de 4 segmentos del Lazo 10 con reflectividad 0.8 .

El segundo escenario simulado se presenta en las figuras 7,8 y 9 . Al igual que antes, se presenta en primer lugar la simulación del funcionamiento del controlador basado en modelo del caso 2, ver figura 7 .
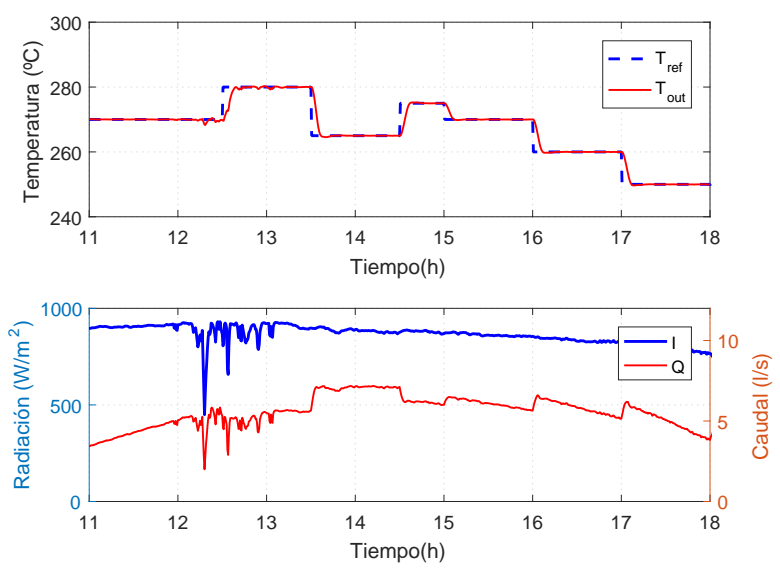

Figura 7: Simulación Caso 2. Seguimiento de setpoint de temperatura de salida de campo.

Las figuras 8 y 9 muestran los resultados de la estimación borrosa de los segmentos de los lazos 4 y 8 del campo con reflectividades 0.74 y 0.79 
respectivamente. Nuevamente se observa que la estimación es bastante fiel al comportamiento real del lazo, tanto en la zona dinámica como en el régimen permanente en torno a los diferentes puntos de operación.
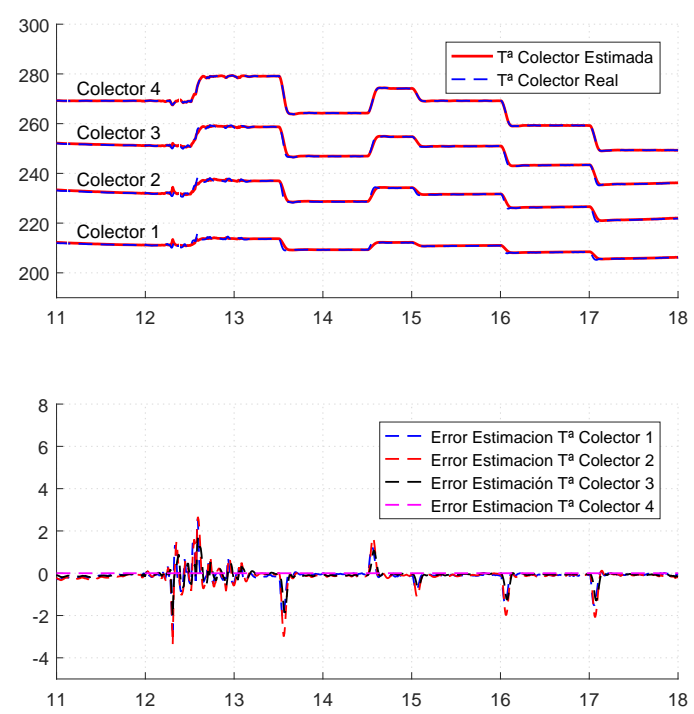

Figura 8: Simulación Caso 2. Etimación de temperaturas de 4 segmentos del Lazo 4 con reflectividad 0.74 .
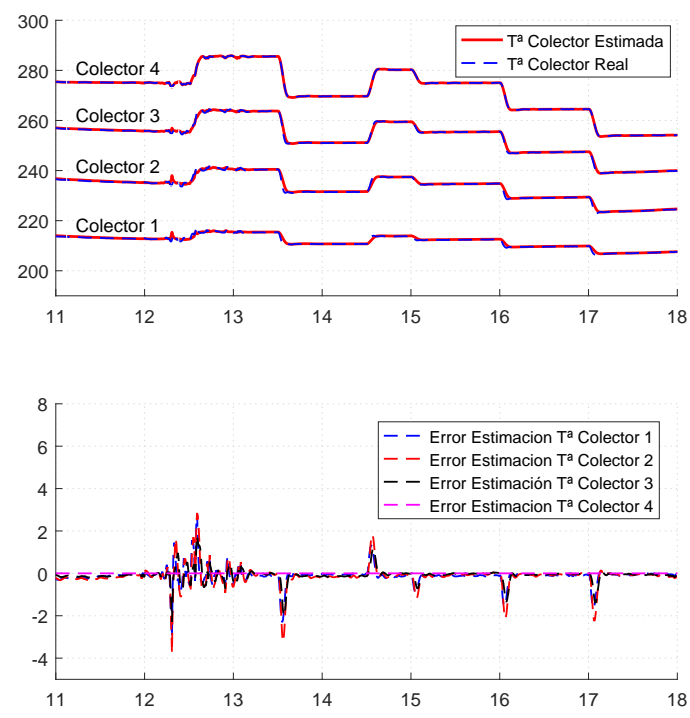

Figura 9: Simulación Caso 2. Etimación de temperaturas de 4 segmentos del Lazo 8 con reflectividad 0.79 .

\section{CONCLUSIONES}

En este trabajo se han desarrollado estimadores borrosos, construidos a partir de datos de operación, mediante técnicas de clustering. Se han usado para la estimación de temperaturas de cuatro segmentos de una planta solar cilindroparabólica. Se han simulados dos casos en los que se presentan diferentes set-points de temperatura a lo largo del día. En estas simulaciones cada lazo tiene una reflectividad diferente. Se han obtenidos resultados estimación muy satisfactorios en lazos con diferentes reflectividades.

\section{Agradecimientos}

Los autores desean agradecer al Ministerio de Economía, Industria y Competitividad español por la financiación del trabajo por el proyecto DPI2016-78338-R.

\section{English summary}

\section{FUZZY ESTIMATION OF A SO- LAR PARABOLIC PLANT)}

\begin{abstract}
The estimation of the unobservable states of a process is important when using control techniques that assume, a priori, these values are known. The controllers based on the state space are very efficient and even more when the dynamics of the process are strongly non-linear. One of the processes with very non-linear dynamics, and in which not all the states are observable, is the case of parabolic trough solar plants. In this work an observer based on Fuzzy Logic is presented to estimate the temperature profiles of the loops that form the solar field
\end{abstract}

Keywords: Fuzzy estimator; Parabolic trough solar plant; Model-based Control.

\section{Referencias}

[1] Pavel Berkhin. Survey of clustering data mining techniques. Technical report, 2002.

[2] E. F. Camacho, M. Berenguel, and F. R. Rubio. Advanced Control of Solar Plants. Springer Science \& Business Media, 1997. 
[3] R. Carmona. Analisis, modelado y control de un campo de colectores solares distribuidos con sistema de seguimiento en un eje. Ph.D. thesis. Universidad de Sevilla, 1985.

[4] S. Chiu. Fuzzy Model Identification based on cluster estimation. Journal of Intelligent Fuzzy Systems, 2:267-278, 1994.

[5] Brian S. Everitt, Sabine Landau, and Morven Leese. Cluster Analysis. Wiley Publishing, 4th edition, 2009.

[6] A. J. Gallego and E. F. Camacho. Adaptative state-space model predictive control of a parabolic-trough field. Control Engineering Practice, 20(9):904 - 911, 2012.

[7] A. J. Gallego, F. Fele, E. F. Camacho, and L. Yebra. Observer-based model predictive control of a parabolic-trough field. Solar Energy, 97:426 - 435, 2013.

[8] A. Ghosh and M. Chakraborty. Hybrid optimized back propagation learning algorithm for multi-layer perceptron. International Journal of Computer Applications, 57, 2012 .

[9] Guzmán. <https://www.nrel.gov/ csp/solarpaces/project_detail.cfm/ projectID=18>, 2018.

[10] A. K. Jain, M. N. Murty, and P. J. Flynn. Data clustering: A review. ACM Comput. Surv., 31(3):264-323, September 1999.

[11] J.S.R. Jang. Anfis: adaptive-network-based fuzzy inference system. IEEE Transactions on Systems, Man, and Cybernetics, 23(3):665-685, May 1993.

[12] Mohammad Karamali and Mahdi Khodabandeh. A distributed solar collector field temperature profile control and estimation using inlet oil temperature and radiation estimates based on iterative extended kalman filter. Renewable Energy, 101:144 - 155, 2017.

[13] Kaxu Solar One. <https://www.nrel.gov/ csp/solarpaces/project_detail.cfm/ projectID=245>, 2018.

[14] A. J. Sánchez, A. J. Gallego, J. M. Escaño, and E. F. Camacho. Temperature homogenization of a solar trough field for performance improvement. Solar Energy. Elsevier., 165C:1-9, 2018.

[15] Solana Generating Station. <https: //www.nrel.gov/csp/solarpaces/ project_detail.cfm/projectID=23>, 2018.
[16] Solar Millennium AG. The construction of the andasol power plants. <http://www. solarmillennium.de/english/archives/ technology/references-and-projects/ andasol-spain/andasol_artikel.html>, 2018.

[17] Tomohiro Takagi and Michio Sugeno. Fuzzy identification of systems and its applications to modeling and control. IEEE Transactions on Systems, Man, and Cybernetics, 15(1):116-132, January 1985.

[18] P. J. Werbos. Beyond Regression: New Tools for Prediction and Analysis in the Behavioral Sciences. PhD thesis, Harvard University, 1974.

[19] Rui Xu and II Wunsch, D. Survey of clustering algorithms. Neural Networks, IEEE Transactions on, 16(3):645-678, May 2005.

[20] R. R. Yager and D. P. Filev. Approximate clustering via the mountain method. IEEE Transactions on Systems, Man, and Cybernetics, 24(8):1279-1284, Aug 1994.

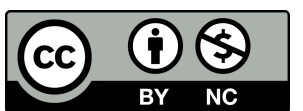

(c) 2018 by the authors. Submitted for possible open access publication under the terms and conditions of the Creative Commons Attribution CC-BY-NC 3.0 license (http://creativecommons.org/licenses/by-nc/3.0/). 